Fetal Diagnosis

and Therapy
Fetal Diagn Ther 2018;43:26-33

DOI: $10.1159 / 000475547$
Received: September 5, 2016

Accepted after revision: April 4, 2017

Published online: September 20, 2017

\title{
Fetal Growth and Placental Growth Factor Umbilical Cord Blood Levels
}

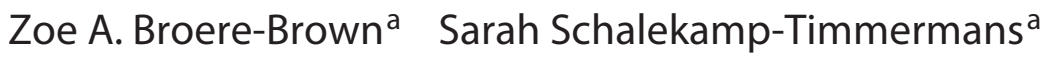 \\ Vincent W.V. Jaddoe ${ }^{b, c}$ Eric A.P. Steegers ${ }^{a}$ \\ Departments of a Obstetrics and Gynecology, ${ }^{\mathrm{b}}$ Epidemiology, and ${ }^{\mathrm{c} P e d i a t r i c s, ~ E r a s m u s ~ M e d i c a l ~ C e n t e r, ~}$ \\ Rotterdam, The Netherlands
}

\section{Keywords}

Placental growth factor · Fetal growth - Birth weight · Fetal growth pattern $\cdot$ Fetal growth restriction

\section{Abstract}

Objective: We assessed whether umbilical cord blood placental growth factor (PIGF) levels at delivery are associated with fetal growth. Methods: From a prospective populationbased cohort study we included 3,461 live singleton births. Fetal growth was assessed by birth weight, fetal growth pattern, and fetal growth restriction (FGR; decrease in growth between the second trimester and birth of $\geq 40$ percentiles). In all analyses the highest PIGF multiple of the median (MoM) quintile was used as the reference category. Results: Umbilical cord PIGF was neither correlated with maternal second-trimester PIGF ( $p=0.08)$ nor placental weight $(p=0.18)$, suggesting that PIGF from umbilical cord blood was of fetal origin. Lower PIGF MoM quintiles were associated with a lower birth weight (lowest quintile -0.60 standard deviation [95\% confidence interval -0.71 to $-0.48, p$ for trend $<0.001]$ ) and a different fetal growth pattern $(p<0.001)$. Finally, lower PIGF MoM quintiles were associated with FGR (lowest quintile odds ratio 2.00 [95\% confidence interval 1.25 to 3.21 , $p$ for trend $<0.001]$ ). Conclusion: Lower umbilical cord PIGF levels are associated with lower birth weight, deviating fetal
\end{abstract}

\section{KARGER}

(C) 2017 S. Karger AG, Basel

E-Mail karger@karger.com

www.karger.com/fdt growth patterns, and a higher odds of FGR. Hence, cord blood PIGF might be a promising biomarker to determine deviations in fetal growth and FGR retrospectively, enabling follow-up of these neonates.

(c) 2017 S. Karger AG, Basel

\section{Introduction}

Placental growth factor (PlGF) is a proangiogenic factor produced by the placenta during pregnancy [1]. Extraplacental tissues associated with a high grade of vascularization, such as the heart and lungs, also produce PlGF [2]. Hence, PlGF is also detectable in nonpregnant women, men, and even in cord blood. To date, the majority of studies have focused predominantly on PlGF in the maternal rather than the fetal circulation [3]. Low levels of maternal PlGF are well known to be associated with placental development and function and subsequently with placenta-mediated pregnancy processes and complications, such as preeclampsia and fetal growth restriction (FGR), demonstrated by a lower birth weight and an increased risk for a neonate to be born small for gestational age (SGA) [4-8]. Interestingly, fetal PlGF levels have been associated with fetal growth. Within our cohort lower levels of umbilical cord PlGF were associated with neonates born SGA [9]. 
However, SGA fetuses constitute a heterogeneous group consisting of fetuses which are constitutionally small and fetuses with FGR resulting in a low birth weight [10-12]. Moreover, neonates could have experienced FGR despite their normal birth weight. Neonates who have experienced FGR may constitute a vulnerable group with an increased risk of accelerated growth and altered cardiovascular outcomes in childhood, irrespective of their birth weight [13, 14]. Identification of FGR neonates is therefore vital.

In this study we aim to assess the association between umbilical cord blood PlGF and fetal growth measured by birth weight, fetal growth pattern, and FGR.

\section{Subjects and Methods}

\section{Study Design and Ethical Approval}

This study was embedded in the Generation R Study, a population-based prospective cohort study from early pregnancy onwards [15]. The study was approved by the Medical Ethics Committee of the Erasmus Medical Center Rotterdam, The Netherlands. Written informed consent was obtained from all participants. For the present study we included women with a live-born singleton with data available concerning fetal growth and cord blood PlGF levels (Fig. 1).

\section{Pregnancy Dating}

Dating by early ultrasonography is vital to ensure accurate pregnancy dating, especially when assessing fetal growth and FGR. Pregnancy dating was performed in early pregnancy using the first ultrasound measurement of either crown-rump length (gestational age until 12 weeks and 5 days of gestation and crown-rump length measurement $<65 \mathrm{~mm}$ ) or biparietal diameter (gestational age from 12 weeks and 5 days of gestation onwards and biparietal diameter $>23 \mathrm{~mm}$ ) [16].

\section{Umbilical Cord Blood PlGF}

Umbilical venous cord blood was sampled immediately after birth and transported to the regional laboratory for processing and storage at $-80^{\circ} \mathrm{C}$. Measurements were performed between 2008 and 2010, and all measurement factors were shown to be stable during long-term storage [17]. Serum levels of PlGF were analyzed using an immunoelectron chemiluminescence assay on the Architect System (Abbott Diagnostics BV). The between-run coefficients of variation for PlGF were $4.7 \%$ at $24 \mathrm{pg} / \mathrm{mL}$ and $3.8 \%$ at 113 $\mathrm{pg} / \mathrm{mL}$ [8]. Umbilical cord blood PlGF is highly dependent of gestational age at birth. Therefore, multiples of the median (MoM) were created for each gestational week separately.

To investigate whether umbilical cord PlGF had a maternal origin, Pearson correlation tests were performed on umbilical cord PlGF levels with first- and second-trimester maternal PlGF levels and placental weight. The Pearson correlation coefficient for umbilical cord PlGF and placental weight was $0.025(p=0.18)$. For maternal PlGF the Pearson correlation coefficients were -0.01 for first-trimester PlGF $(p=0.69)$ and 0.03 for second-trimester PlGF ( $p=0.08)$, suggesting that fetal PlGF levels instead of maternal PlGF levels were measured in umbilical blood.

Fetal Growth and PlGF Cord Blood Levels

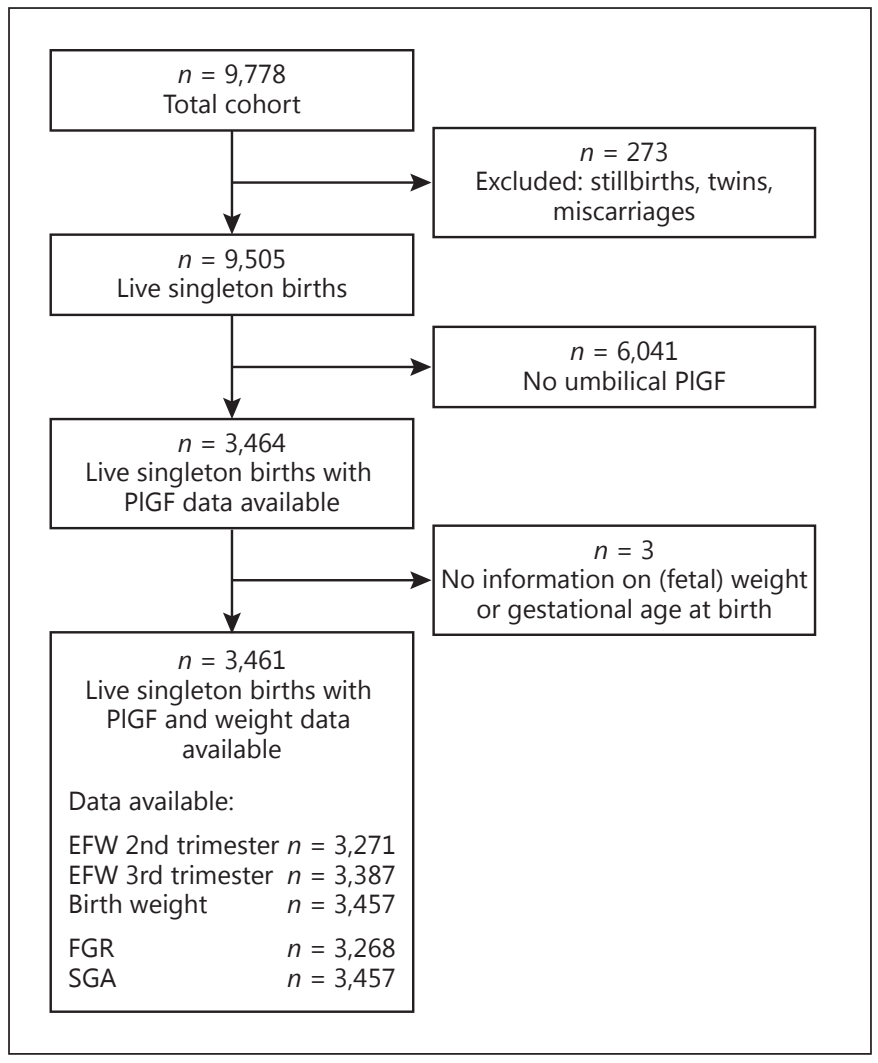

Fig. 1. Flowchart of the selection process. EFW, estimated fetal weight; FGR, fetal growth restriction; PlGF, placental growth factor; SGA, small for gestational age.

\section{Fetal Growth}

To assess estimated fetal weight (EFW), ultrasound examinations were performed in the second (median 20.5 weeks of gestation, 90\% range 18.9-22.8) and third trimester (median 30.4 weeks of gestation, $90 \%$ range $28.8-32.4$ ) of pregnancy. Fetal biometry (head circumference $[\mathrm{HC}]$, abdominal circumference $[\mathrm{AC}]$, and femur length $[\mathrm{FL}]$ ) was measured transabdominally. EFW was calculated using the formula of Hadlock with the parameters AC, HC, and FL (in cm): $\mathrm{EFW}=10^{* *}[1.326-(0.00326 \times \mathrm{AC} \times \mathrm{FL})+$ $(0.0107 \times \mathrm{HC})+(0.0438 \times \mathrm{AC})+(0.158 \times \mathrm{FL})][18]$. Ultrasound examinations were performed using Aloka ${ }^{\circledR}$ model SSD-1700 (Tokyo, Japan) or the ATL-Philips ${ }^{\circledR}$ Model HDI 5000 (Seattle, WA, USA). Gestational age at birth and birth weight were obtained from midwives and hospital registries. SGA was defined as a gestational and fetal sex-adjusted birth weight below the fifth percentile based on charts derived from our cohort [16]. Five definitions were used to define FGR: a decrease in growth of at least 30, 35, 40, 45 , or 50 percentiles, respectively between the second trimester and birth. Choosing cutoffs to define FGR may lead to misclassification. For a fetus to be able to deviate from its growth curve with 40 percentiles, the initial EFW in the second trimester of pregnancy should by definition have been above the 40th percentile. Hereby fetuses with an EFW below the 40th percentile cannot be classified as FGR and are thus classified as not growth restricted. Therefore, in an additional analysis, we classified all fetuses with

Fetal Diagn Ther 2018;43:26-33 27 
an EFW in the 2nd trimester below the 40th percentile and with a maximum decrease in growth (birth weight below the 1st percentile) as FGR, without any differences in results.

\section{Covariates}

We obtained information on maternal age, ethnicity, educational level, parity, smoking during pregnancy, and folic acid intake by questionnaire at enrolment. At intake height $(\mathrm{cm})$ and weight $(\mathrm{kg})$ were measured without shoes and heavy clothing and the body mass index (BMI) was calculated. Preeclampsia was defined as the development of a systolic blood pressure $\geq 140 \mathrm{~mm} \mathrm{Hg}$ and/or a diastolic blood pressure $\geq 90 \mathrm{~mm} \mathrm{Hg}$ after 20 weeks of gestation plus the presence of proteinuria $(\geq 0.3 \mathrm{~g}$ in a 24 -h urine specimen or $2+$ or greater $[1 \mathrm{~g} / \mathrm{L}]$ on a voiced specimen, or $1+$ or greater $[0.3 \mathrm{~g} / \mathrm{L}]$ on a catheterized specimen) in previously normotensive women according to the former International Society for the Study of Hypertension in Pregnancy criteria [19].

\section{Statistical Analyses}

To explore nonlinearity, PlGF MoM was categorized into quintiles: first quintile (PlGF MoM >1.27), second quintile (PlGF MoM $<1.27$ and $>1.08$ ), third quintile (PlGF MoM $<1.08$ and $>0.93$ ), fourth quintile (PlGF MoM $<0.93$ and $>0.78$ ), and fifth quintile (PlGF MoM <0.78).

To test the differences in baseline characteristics between the five PlGF MoM quintiles, ANOVA, Mann-Whitney U, and $\chi^{2}$ tests were performed. Then linear regression analyses were performed to relate cord blood PlGF MoMs to birth weight. To explore growth trajectories between different PlGF MoM categories, unbalanced repeated-measurements regression models with an unstructured covariance structure were performed. The mixed-model procedure was used with (estimated) weight expressed in standard deviation as a repeated outcome measure. These regression models take the correlation between repeated measurements of the same subject into account. Moreover, they have an optimal use of available measurements by allowing for incomplete outcome data. Then logistic regression models were used to analyze the associations of umbilical cord blood PlGF MoMs with FGR.

Basic models were adjusted for fetal sex and ethnicity. Confounder models were additionally adjusted for maternal age, educational level, parity, smoking during pregnancy, and folic acid intake. We selected these confounders on the basis of their associations with both the exposure and the outcomes of interest or a change in effect estimate of $>10 \%$. We considered the confounder model to be the main model. Since angiogenic levels are disturbed in case of maternal preeclampsia, a third model was used in which we additionally adjusted for the presence of preeclampsia (preeclampsia model).

Effect modification on the multiplicative scale was tested to exclude the possibility that the effect of low cord blood PlGF MoM on fetal growth depends on fetal sex, smoking, parity, maternal BMI at intake, and SGA. If $p<0.10$ was fulfilled, regression analyses were performed in strata of that specific variable.

For all analyses, the percentages of missing values of covariates were $<20 \%$. We imputed missing data of the covariates by using multiple imputations [20]. Ten datasets were created and analyzed together. Statistical analyses were performed using the Statistical Package of Social Sciences version 21.0 for Windows (SPSS Inc., Chicago, IL, USA) or the Statistical Analysis System version 9.3 (SAS Institute Inc., Gary, NC, USA).

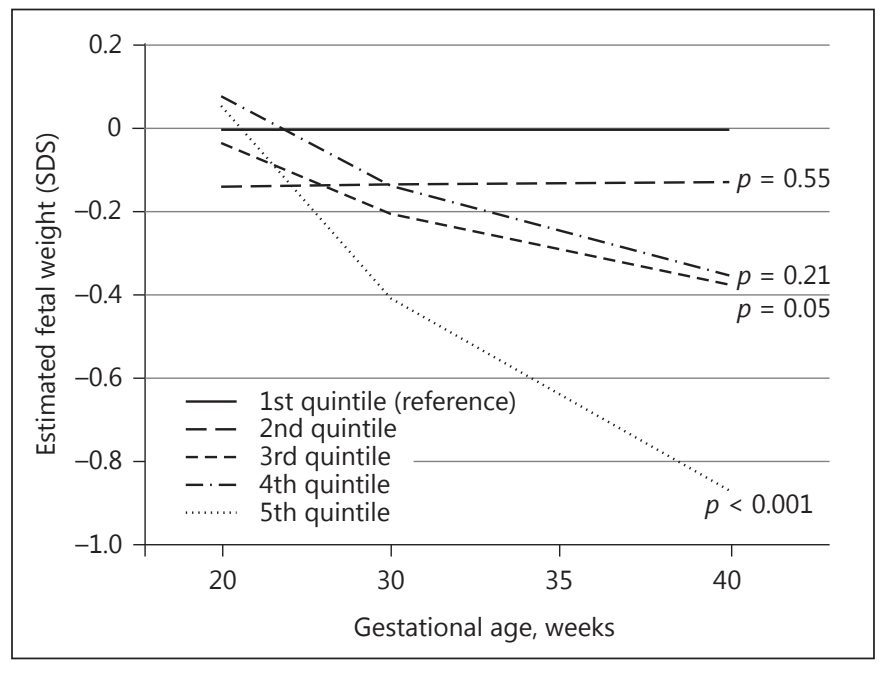

Fig. 2. Associations between placental growth factor and fetal growth pattern. Results are based on repeated-measurements regression models and reflect the differences in gestational age-adjusted standard deviation scores at 20,30,35, and 40 weeks of gestation. The highest quintile of placental growth factor is the reference category and presented as the zero line in the graph. The model was adjusted for fetal sex, ethnicity, maternal age, educational level, parity, smoking during pregnancy, and folic acid intake. SDS, standard deviation score.

\section{Results}

The baseline characteristics of the total study population are presented in Table 1. Stratification between the different PlGF MoM quintiles showed that mothers of neonates with lower PlGF MoMs were more often nulliparous $(p<0.001)$ and more often had preeclampsia $(p=0.007)$. The neonates themselves had a lower birth weight $(p<0.001)$.

The results regarding birth weight (standard deviation score) are depicted in Table 2. All quintiles, with the exception of the second quintile, were associated with lower birth weights, with a $p$ for trend $<0.001$ in all models compared to the first quintile.

The results from repeated measurements showed a different growth pattern in the lowest PlGF MoM quintile compared to the highest quintile (Fig. 2, $p<0.001$ ). This resulted in a lower EFW and birth weight. All other quintiles had lower EFWs and lower birth weights, however with a similar growth pattern compared with the highest PlGF MoM quintile.

The results regarding the clinical outcome FGR are depicted in Table 3. Again similar results towards lower PlGF MoMs versus decreased fetal growth were observed,
28

Fetal Diagn Ther 2018;43:26-33

DOI: $10.1159 / 000475547$
Broere-Brown/Schalekamp-

Timmermans/Jaddoe/Steegers 
Table 1. Baseline characteristics of the total study population

\begin{tabular}{|c|c|c|c|c|c|c|c|}
\hline & $\begin{array}{l}\text { Study population } \\
(n=3,461)\end{array}$ & $\begin{array}{l}\text { Highest quintile } \\
(n=696)\end{array}$ & $\begin{array}{l}\text { Second quintile } \\
(n=695)\end{array}$ & $\begin{array}{l}\text { Third quintile } \\
(n=697)\end{array}$ & $\begin{array}{l}\text { Fourth quintile } \\
(n=678)\end{array}$ & $\begin{array}{l}\text { Lowest quintile } \\
(n=695)\end{array}$ & $\begin{array}{l}p \\
\text { value }\end{array}$ \\
\hline Maternal age & $29.6 \pm 5.2$ & $29.6 \pm 5.1$ & $29.5 \pm 5.3$ & $29.6 \pm 5.1$ & $29.5 \pm 5.2$ & $29.7 \pm 5.4$ & 0.96 \\
\hline \multicolumn{8}{|l|}{ Anthropometrics } \\
\hline Height, cm & $167.7 \pm 7.4$ & $168.0 \pm 7.6$ & $167.9 \pm 7.1$ & $167.8 \pm 7.1$ & $167.6 \pm 7.7$ & $167.0 \pm 7.3$ & 0.1 \\
\hline Weight, kg & $69.4 \pm 12.9$ & $69.4 \pm 12.8$ & $69.8 \pm 13.0$ & $68.9 \pm 13.0$ & $69.7 \pm 13.1$ & $69.0 \pm 12.7$ & 0.6 \\
\hline $\mathrm{BMI}$ & $23.8(19.3-33.2)$ & $23.8(19.3-33.2)$ & $23.9(19.5-33.3)$ & $23.4(19.0-33.1)$ & $23.8(19.5-33.4)$ & $24.0(19.4-33.4)$ & 0.31 \\
\hline \multicolumn{7}{|l|}{ Ethnicity } & 0.94 \\
\hline Western & $2,047(61.6 \%)$ & $413(61.9 \%)$ & $414(62.3 \%)$ & $418(62.3 \%)$ & 398 (61.1\%) & $403(60.3 \%)$ & \\
\hline Non-Western & $1,276(38.4 \%)$ & $254(39.1 \%)$ & $251(37.7 \%)$ & $253(37.7 \%)$ & $253(39.9 \%)$ & $265(39.7 \%)$ & \\
\hline \multicolumn{7}{|l|}{ Educational level } & 0.08 \\
\hline Low & $391(11.3 \%)$ & $77(11.1 \%)$ & $88(12.7 \%)$ & $79(11.3 \%)$ & $83(12.2 \%)$ & $65(9.4 \%)$ & \\
\hline Middle & $1,589(46.1 \%)$ & $326(46.8 \%)$ & $306(44.0 \%)$ & $309(44.3 \%)$ & $311(45.9 \%)$ & $346(49.8 \%)$ & \\
\hline High & $1,468(42.6 \%)$ & $293(42.1 \%)$ & $301(43.3 \%)$ & $309(44.3 \%)$ & $284(41.9 \%)$ & $284(40.9 \%)$ & \\
\hline \multicolumn{7}{|l|}{ Smoking habits } & 0.46 \\
\hline No & $2,470(71.6 \%)$ & 499 (71.7\%) & $506(72.8 \%)$ & $486(69.7 \%)$ & $484(71.4 \%)$ & $500(71.9 \%)$ & \\
\hline Yes, stopped & $291(8.4 \%)$ & $50(7.2 \%)$ & $58(8.3 \%)$ & $64(9.2 \%)$ & $68(10.0 \%)$ & $55(7.9 \%)$ & \\
\hline Yes, continued & $688(19.9 \%)$ & $147(21.1 \%)$ & $131(18.8 \%)$ & $147(21.1 \%)$ & $126(18.6 \%)$ & $140(20.1 \%)$ & \\
\hline \multicolumn{7}{|l|}{ Folic acid use } & 0.18 \\
\hline No & $990(28.7 \%)$ & $191(27.4 \%)$ & $201(28.9 \%)$ & $194(27.8 \%)$ & $205(30.2 \%)$ & $192(27.6 \%)$ & \\
\hline Yes, before 10 weeks & $1,099(31.8 \%)$ & $218(31.3 \%)$ & $229(32.9 \%)$ & $219(31.4 \%)$ & $221(32.6 \%)$ & $229(32.9 \%)$ & \\
\hline Yes, preconception start & $1,360(39.4 \%)$ & $287(41.2 \%)$ & $265(38.1 \%)$ & $284(40.7 \%)$ & $252(37.2 \%)$ & $274(39.4 \%)$ & \\
\hline Nulliparous & $1,942(56.3 \%)$ & $320(46.0 \%)$ & $318(45.8 \%)$ & $399(57.2 \%)$ & $421(62.1 \%)$ & $490(70.5 \%)$ & $<0.001$ \\
\hline Preeclampsia & $42(1.3 \%)$ & $5(0.7 \%)$ & $5(0.7 \%)$ & $5(0.7 \%)$ & $10(1.5 \%)$ & $17(2.4 \%)$ & 0.007 \\
\hline \multicolumn{8}{|l|}{ GA at sonography, weeks } \\
\hline Second trimester & $20.5(18.9-22.8)$ & $20.5(19.0-22.9)$ & $20.5(18.8-22.7)$ & $20.5(18.9-22.8)$ & $20.5(18.8-22.8)$ & $20.5(18.9-22.9)$ & 0.65 \\
\hline Third trimester & $30.4(28.8-32.3)$ & $30.4(28.8-32.3)$ & $30.4(28.8-32.3)$ & $30.3(28.9-32.3)$ & $30.3(28.7-32.2)$ & $30.4(28.6-32.6)$ & 0.72 \\
\hline GA at birth, weeks & $40.1(37.4-42.0)$ & $40.1(37.1-42.1)$ & $40.1(37.6-42.0)$ & $40.0(37.4-42.0)$ & $40.1(37.4-42.0)$ & $40.3(37.3-42.2)$ & 0.28 \\
\hline EFW 2nd trimester, g & $382 \pm 94$ & $384 \pm 91$ & $381 \pm 95$ & $385 \pm 98$ & $380 \pm 95$ & $383 \pm 93$ & 0.87 \\
\hline EFW 3rd trimester, g & $1,614 \pm 255$ & $1,619 \pm 241$ & $1,627 \pm 274$ & $1,611 \pm 38$ & $1,603 \pm 253$ & $1,612 \pm 265$ & 0.52 \\
\hline Birth weight, g & $3,458 \pm 500$ & $3,556 \pm 535$ & $3,551 \pm 463$ & $3,451 \pm 471$ & $3,431 \pm 475$ & $3,298 \pm 509$ & $<0.001$ \\
\hline PlGF MoM & $1.00(0.58-1.75)$ & $1.47(1.29-5.09)$ & $1.16(1.08-1.26)$ & $1.00(0.93-1.07)$ & $0.86(0.79-0.92)$ & $0.64(0.41-0.77)$ & $<0.001$ \\
\hline
\end{tabular}

Table 2. Associations between cord blood PlGF and birth weight

\begin{tabular}{|c|c|c|c|c|c|}
\hline Basic model & & Confounde & & Preeclamps & \\
\hline$\beta(95 \% \mathrm{CI})$ & $p$ value & $\beta(95 \% \mathrm{CI})$ & $p$ value & $\beta(95 \% \mathrm{CI})$ & $p$ value \\
\hline
\end{tabular}

Birth weight (SDS)

PlGF MoM 1st quintile reference

PlGF MoM 2nd quintile $-0.02(-0.12$ to 0.09$)$

PlGF MoM 3rd quintile $-0.22(-0.33$ to -0.12$)$

PlGF MoM 4th quintile $\quad-0.29(-0.39$ to -0.18$)$

PlGF MoM 5th quintile $\quad-0.60(-0.71$ to -0.48$)$

$p$ for trend reference

\begin{tabular}{llc}
\multicolumn{1}{c}{0.74} & $-0.02(-0.12$ to 0.08$)$ & 0.70 \\
$<0.001$ & $-0.19(-0.29$ to -0.08$)$ & $<0.001$ \\
$<0.001$ & $-0.24(-0.34$ to -0.13$)$ & $<0.001$ \\
$<0.001$ & $-0.50(-0.61$ to -0.38$)$ & $<0.001$ \\
$<0.001$ & $p$ for trend & $<0.001$
\end{tabular}

reference

$\begin{array}{lc}-0.02(-0.12 \text { to } 0.08) & 0.72 \\ -0.19(-0.29 \text { to }-0.08) & <0.001 \\ -0.23(-0.33 \text { to }-0.13) & <0.001 \\ -0.48(-0.60 \text { to }-0.37) & <0.001 \\ p \text { for trend } & <0.001\end{array}$

\section{reference}

$-0.19(-0.29$ to -0.08$)$

$-0.23(-0.33$ to -0.13$)$

-0.48 ( -0.60 to -0.37$)$

$<0.001$

Values are regression coefficient (95\% CI) and are based on linear regression models. Basic model: adjusted for fetal sex and ethnicity. Confounder model: basic model additionally adjusted for maternal age, educational level, parity, smoking, and folic acid intake. Preeclamptic model: confounder model additionally adjusted for maternal preeclampsia. CI, confidence interval; MoM, multiple of the median; PlGF, placental growth factor; SDS, standard deviation score. 
Table 3. Associations between cord blood placental growth factor and FGR

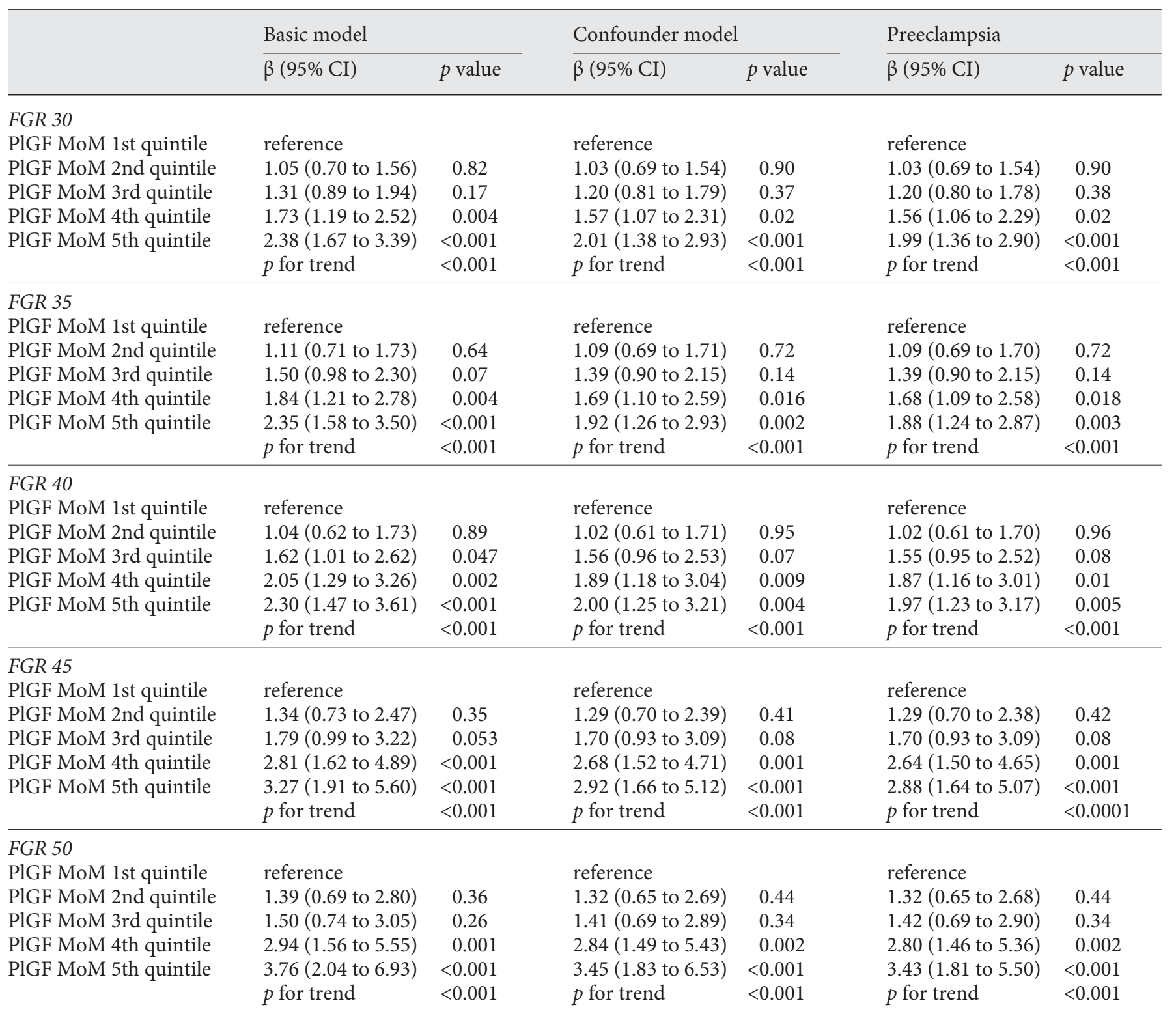

Values are regression coefficient (95\% CI) and are based on linear regression models. Basic model: adjusted for fetal sex and ethnicity. Confounder model: basic model additionally adjusted for maternal age, educational level, parity, smoking, and folic acid intake. Preeclampsia model: confounder model additionally adjusted for maternal preeclampsia. CI, confidence interval; FGR, fetal growth restriction; MoM, multiple of the median; PlGF, placental growth factor.

with odds ratios up to 3.45 (95\% confidence interval 1.83 to 6.53) for the fifth quintile for FGR with a decrease in growth of $>50$ percentiles.

Interaction analyses showed no effect modification of fetal sex, smoking, parity, maternal BMI at intake, and SGA on all outcomes.

\section{Discussion}

\section{Principal Findings}

This study showed that umbilical cord blood PlGF is associated with fetal growth, with consistent results for birth weight, growth pattern, and the outcome FGR. The
Fetal Diagn Ther 2018;43:26-33
DOI: $10.1159 / 000475547$
Broere-Brown/SchalekampTimmermans/Jaddoe/Steegers 
lower PlGF levels, the stronger the observed effect estimates.

\section{Strengths and Limitations}

The main strength of this study was the extensive prospective data collection on fetal growth and environmental influences. This enabled us to adjust for multiple confounding factors and to investigate the effects of fetal growth in a large sample of 3,461 participants.

The response rate at baseline for participation in the Generation R Study was $61 \%$. Selective participations in this study did occur since pregnant women who participated were generally higher educated, more healthy, and more frequently of European origin than those who did not participate. Data on umbilical cord blood PlGF were available in $36.4 \%$ of our total live singleton births. The nonresponse at baseline would lead to biased effect estimates if the associations were different between those included and those not included in the analyses. However, given the prospective nature of the study, this seems unlikely. Nevertheless it might have led to the selection of a more healthy population, which affects the generalizability of our results.

For the purpose of this study we included only live singleton births. Therefore, intrauterine fetal deaths with or without FGR were excluded. As a consequence, the included pregnancies constitute a relatively healthy and homogenous study population.

In this study, we focused on a true deviation of fetal growth rather than on other parameters such as perinatal outcomes, Doppler parameters, or placental function. SGA was defined as a gestational and fetal sex-adjusted birth weight below the fifth percentile based on charts derived from our cohort. This cutoff was chosen since the fifth percentile of our cohort is more comparable with the cutoff of the tenth percentile used in general clinical practice, compared with the tenth percentile of our cohort.

\section{Interpretation}

One of the key findings of this study is that not only the lowest quintile of cord blood PlGF was associated with fetal growth, but that also associations for higher quintiles were found and that clear trends were visible. Although this is an observational study and causality can therefore not be proven, one of the most important criteria for causation according to the Bradford Hill criteria is a biological gradient [21]. In our study it is clear that a clear trend was visible in the association between neonatal PlGF levels and fetal growth parameters. Moreover,

Fetal Growth and PlGF Cord Blood Levels the effect sizes are substantial and consistent despite adjustment for multiple confounding factors.

Fetal cord blood may contain PlGF of fetal and maternal or placental origin since PlGF crosses the placenta. However, very low correlations were found between umbilical cord blood PlGF levels on the one hand and maternal PlGF levels and placental weight on the other. Furthermore, there were hardly any differences in effect estimates of the confounder models and the preeclampsia models. This implies that the vast majority of PlGF measured in umbilical cord blood is of fetal origin. Unfortunately no maternal PlGF levels at the end of pregnancy were available within our cohort. During pregnancy, PlGF reaches its peak around 31 weeks of gestation, after which it declines [7, 22]. Around the time of delivery, levels of maternal PIGF are comparable with levels around 19 weeks of gestation. Correlation coefficients might therefore not be drastically different even if term maternal PlGF at term had been available. This is confirmed by one study in which there was a 10 -fold difference for PlGF in the maternal and fetal circulation during delivery and without a significant correlation between the two [23]. It is suggested that the umbilical artery PlGF would be a better representation of the fetal circulation compared to the umbilical vein PlGF which we used in our study. Some studies investigated PlGF in both the umbilical vein as well as arteries in SGA neonates compared with appropriate for gestational age neonates and considered the umbilical vein as a representation of the placental compartment [24]. They observed differences in PIGF levels between SGA and appropriate for gestational age neonates in the umbilical vein, but not in the umbilical artery, and concluded that fetal PlGF was not altered in SGA. However, if the umbilical vein indeed is a representation of the placental compartment, a significant correlation would be expected between umbilical vein PlGF and maternal PlGF or measures of placental function such as placental weight. In our study these associations were not present, from which we conclude that also the umbilical vein is representative for the fetal circulation.

In the current literature, the clinical outcome FGR is often defined as SGA [25]. However, FGR is not necessarily synonymous with SGA. FGR fetuses may experience a failure to reach their biological growth potential because of a pathological slowdown in the fetal growth pace. Recently, the Delphi study also established a definition for FGR by expert consensus, acknowledging the significant difference between FGR and SGA [25]. The definitions of both early and late FGR were based on

Fetal Diagn Ther 2018;43:26-33 DOI: $10.1159 / 000475547$ 
measurements taken at one single point in time. Although crossing centiles for AC or EFW were allowed in the definition for late FGR, it must be combined with another criterion. We, on the other hand, primarily and solely focused on deviating growth patterns. Since there is no international consensus on how much a fetal growth curve needs to deviate before it can be designated as FGR, we used several cutoffs of a decrease in growth between the second trimester of pregnancy and birth. In previous studies, FGR fetuses without SGA were not the subject of research. Nevertheless, this might be a vulnerable group with increased risks of adverse health in later life. The current study shows an association between fetal PlGF and the clinical outcome FGR. These associations were not only found for the severe growth restricted fetuses, i.e., a decrease of $\geq 50$ percentiles, but also for the mildly growth-restricted fetuses, i.e., a decrease of $\geq 30$ percentiles. Future research has to focus on the potential of fetal PlGF to identify those neonates at risk for adverse childhood outcomes.

Since little research has been performed on neonatal PlGF, not much is known about the exact origin of neonatal PlGF. PlGF is not only expressed by the placenta, but also by tumor cells, endothelial cells, and inflammatory cells [26]. Especially endothelial cells could be a potential source of PlGF within the neonate. PlGF exerts a strong effect upon blood vessel growth and maturation and has direct proangiogenic effects on endothelial cells [27]. Within cardiovascular disease, endothelial function is crucial and often precedes clinical manifestations [28, 29]. This is of importance when taking the Development and Origins of Health and Disease theory into consideration. According to this theory, the unborn fetus can modify its own development such that it will be prepared for survival in an environment in which recourses are likely to be short. Although these adaptations may be beneficial for short-term survival, they may have adverse consequences at delivery or in later life [30]. Since FGR fetuses have an increased risk of developing cardiac diseases in later life, low cord blood levels of PIGF could well be one of the first signs of this increased risk. Nevertheless, we should consider the possibility that small fetuses produce less PlGF. In that case the underlying mechanisms of the fetuses being small cause the potential adverse outcomes in later life associated with FGR rather than PlGF itself. However, this seems unlikely considering the incidence of preeclampsia as shown in Table 1, which differs considerably across the different PIGF quintiles.

\section{Conclusion}

This study demonstrates that umbilical cord blood PlGF is associated with birth weight, fetal growth pattern, and FGR. Fetuses born with FGR are often born with a normal birth weight, but nevertheless constitute a vulnerable group with an increased risk for cardiovascular diseases in later life. Identification of this vulnerable group enables follow-up in the postnatal period, preventing possible adverse events in later life. Future research is needed to investigate whether fetal PlGF can be used to identify those fetuses at risk for adverse childhood outcomes.

\section{Acknowledgments}

The Generation R Study is being conducted by Erasmus Medical Center in close collaboration with the School of Law and the Faculty of Social Sciences of Erasmus University Rotterdam, the Municipal Health Service, Rotterdam Area, the Rotterdam Homecare Foundation, and the Stichting Trombosedienst and Artsenlaboratorium Rijnmond (Rotterdam, The Netherlands). It was made possible by financial support from Erasmus Medical Center, Erasmus University Rotterdam, the Netherlands Organization for Health Research and Development, the Netherlands Organization for Scientific Research, the Ministry of Health, Welfare, and Sports, and the Ministry of Youth and Families. We gratefully acknowledge the contributions of the general practitioners, hospitals, midwives, and pharmacies in Rotterdam.

\section{Disclosure Statement}

The authors have no conflicting interests. Dr. V.W.V. Jaddoe received additional grants from the Netherlands Organization for Health Research and Development (grants 90700303 and 916.10159).

\section{Author Contributions}

Z.A. Broere-Brown analyzed the data and wrote the article. S. Schalekamp-Timmermans contributed to the design of the article and assisted with the analyses and writing of the article. V.W.V. Jaddoe contributed to the design of the study, the interpretation of the data, and revisions and gave input at all stages of the study. E.A.P. Steegers contributed to the design of the study, the writing of the article, interpretation of the data, and revisions and gave input at all stages of the study. All authors have approved the final version of the manuscript.
Broere-Brown/SchalekampTimmermans/Jaddoe/Steegers 


\section{References}

1 Maglione D, Guerriero V, Viglietto G, DelliBovi P, Persico MG: Isolation of a human placenta cDNA coding for a protein related to the vascular permeability factor. Proc Natl Acad Sci USA 1991;88:9267-9271.

2 Andraweera PH, Dekker GA, Roberts CT: The vascular endothelial growth factor family in adverse pregnancy outcomes. Hum Reprod Update 2012; 18:436-457.

3 Triunfo S, Lobmaier S, Parra-Saavedra M, Crovetto F, Peguero A, Nadal A, Gratacos E, Figueras F: Angiogenic factors at diagnosis of late-onset small-for-gestational age and histological placental underperfusion. Placenta 2014;35:398-403.

4 Levine RJ, Maynard SE, Qian C, Lim KH, England LJ, Yu KF, Schisterman EF, Thadhani R, Sachs BP, Epstein FH, Sibai BM, Sukhatme VP, Karumanchi SA: Circulating angiogenic factors and the risk of preeclampsia. N Engl J Med 2004;350:672-683.

5 Taylor RN, Grimwood J, Taylor RS, McMaster MT, Fisher SJ, North RA: Longitudinal serum concentrations of placental growth factor: evidence for abnormal placental angiogenesis in pathologic pregnancies. Am J Obstet Gynecol 2003;188:177-182.

6 Tidwell SC, Ho HN, Chiu WH, Torry RJ, Torry DS: Low maternal serum levels of placenta growth factor as an antecedent of clinical preeclampsia. Am J Obstet Gynecol 2001;184: 1267-1272.

7 Sundrani D, Khot V, Pisal H, Mehendale S, Wagh G, Joshi A, Joshi S: Gestation dependent changes in angiogenic factors and their associations with fetal growth measures in normotensive pregnancy. PLoS One 2013; 8:e54153.

8 Coolman M, Timmermans S, de Groot CJ Russcher H, Lindemans J, Hofman A, GeurtsMoespot AJ, Sweep FC, Jaddoe VV, Steegers EA: Angiogenic and fibrinolytic factors in blood during the first half of pregnancy and adverse pregnancy outcomes. Obstet Gynecol 2012;119:1190-1200.

9 Bergen NE, Bouwland-Both MI, SteegersTheunissen RP, Hofman A, Russcher H, Lindemans J, Jaddoe VW, Steegers EA: Early pregnancy maternal and fetal angiogenic factors and fetal and childhood growth: the Generation R Study. Hum Reprod 2015;30:13021313.

10 Alberry M, Soothill P: Management of fetal growth restriction. Arch Dis Child Fetal Neonatal Ed 2007;92:F62-F67.
11 Illanes S, Soothill P: Management of fetal growth restriction. Semin Fetal Neonatal Med 2004;9:395-401.

12 Figueras F, Gratacos E: Stage-based approach to the management of fetal growth restriction. Prenat Diagn 2014;34:655-659.

13 Crispi F, Bijnens B, Figueras F, Bartrons J, Eixarch E, Le Noble F, Ahmed A, Gratacos E: Fetal growth restriction results in remodeled and less efficient hearts in children. Circulation 2010;121:2427-2436.

14 Figueras F, Oros D, Cruz-Martinez R, Padilla N, Hernandez-Andrade E, Botet F, CostasMoragas C, Gratacos E: Neurobehavior in term, small-for-gestational age infants with normal placental function. Pediatrics 2009; 124:e934-e941

15 Jaddoe VW, van Duijn CM, Franco OH, van der Heijden AJ, van Iizendoorn $\mathrm{MH}$, de Jongste JC, van der Lugt A, Mackenbach JP, Moll HA, Raat H, Rivadeneira F, Steegers EA, Tiemeier H, Uitterlinden AG, Verhulst FC, Hofman A: The Generation R Study: design and cohort update 2012. Eur J Epidemiol 2012;27: 739-756.

16 Verburg BO, Steegers EA, De Ridder M, Snijders RJ, Smith E, Hofman A, Moll HA, Jaddoe VW, Witteman JC: New charts for ultrasound dating of pregnancy and assessment of fetal growth: longitudinal data from a populationbased cohort study. Ultrasound Obstet Gynecol 2008;31:388-396.

17 Law LW, Sahota DS, Chan LW, Chen M, Lau TK, Leung TY: Effect of long-term storage on placental growth factor and fms-like tyrosine kinase 1 measurements in samples from pregnant women. J Matern Fetal Neonatal Med 2010;23:1475-1480.

18 Hadlock FP, Harrist RB, Sharman RS, Deter RL, Park SK: Estimation of fetal weight with the use of head, body, and femur measurements - a prospective study. Am J Obstet Gynecol 1985;151:333-337.

19 Report of the National High Blood Pressure Education Program Working Group on High Blood Pressure in Pregnancy. Am J Obstet Gynecol 2000;183:S1-S22.

20 Sterne JA, White IR, Carlin JB, Spratt M, Royston P, Kenward MG, Wood AM, Carpenter JR: Multiple imputation for missing data in epidemiological and clinical research: potential and pitfalls. BMJ 2009;338:b2393.

21 Hill AB: The environment and disease: association or causation? Proc R Soc Med 1965;58: 295-300.
22 Tsiakkas A, Duvdevani N, Wright A, Wright D, Nicolaides KH: Serum placental growth factor in the three trimesters of pregnancy: effects of maternal characteristics and medical history. Ultrasound Obstet Gynecol 2015;45: 591-598.

23 Algeri P, Ornaghi S, Bernasconi DP, Cappellini F, Signorini S, Brambilla P, Urban G, Vergani P: Feto-maternal correlation of PTX3, sFlt-1 and PlGF in physiological and preeclamptic pregnancies. Hypertens Pregnancy 2014;33:360-370.

24 Wallner W, Sengenberger R, Strick R, Strissel PL, Meurer B, Beckmann MW, Schlembach $\mathrm{D}$ : Angiogenic growth factors in maternal and fetal serum in pregnancies complicated by intrauterine growth restriction. Clin Sci (Lond) 2007;112:51-57.

25 Gordijn SJ, Beune IM, Thilaganathan B, Papageorghiou A, Baschat AA, Baker PN, Silver RM, Wynia K, Ganzevoort W: Consensus definition of fetal growth restriction: a Delphi procedure. Ultrasound Obstet Gynecol 2016; 48:333-339.

26 Cao Y: Positive and negative modulation of angiogenesis by VEGFR1 ligands. Sci Signal 2009;2:re1.

27 Carmeliet P, Moons L, Luttun A, Vincenti V, Compernolle V, De Mol M, Wu Y, Bono F, Devy L, Beck H, Scholz D, Acker T, DiPalma T, Dewerchin M, Noel A, Stalmans I, Barra A, Blacher S, VandenDriessche T, Ponten A, Eriksson U, Plate KH, Foidart JM, Schaper W, Charnock-Jones DS, Hicklin DJ, Herbert JM, Collen D, Persico MG: Synergism between vascular endothelial growth factor and placental growth factor contributes to angiogenesis and plasma extravasation in pathological conditions. Nat Med 2001;7:575-583.

28 Hadi HA, Carr CS, Al Suwaidi J: Endothelial dysfunction: cardiovascular risk factors, therapy, and outcome. Vasc Health Risk Manag 2005;1:183-198.

29 Gidullin NS, Valeeva KF, Gassanov N, Zagidullin SZ: Value of endothelial dysfunction in cardiovascular diseases and methods of its correction with drugs (in Russian). Kardiologiia 2010;50:54-60.

30 Barker DJ, Osmond C: Infant mortality, childhood nutrition, and ischaemic heart disease in England and Wales. Lancet 1986;1: 1077-1081. 